\title{
Educação permanente aplicada aos profissionais do setor de hemoterapia: uma
}

\section{revisão integrativa}

\author{
Permanent education applied to professionals in the hemotherapy sector: an integrating review
}

Educación permanente aplicada a profesionales del sector de la hemoterapia: una revisión

integrativa

Recebido: 08/01/2021 | Revisado: 11/01/2021 | Aceito: 15/01/2021 | Publicado: 19/01/2021

\author{
Milena Marques Dias \\ ORCID: https://orcid.org/0000-0002-8505-2146 \\ Universidade Federal Fluminense, Brasil \\ E-mail:mdias.farm@gmail.com \\ Benedito Carlos Cordeiro \\ ORCID: https://orcid.org/0000-0001-6387-511X \\ Universidade Federal Fluminense, Brasil \\ E-mail: bcordeiro@id.uff.br \\ Giselle de Fatima Gonçalves \\ ORCID: https://orcid.org/0000-0002-9892-1654 \\ Universidade Federal do Rio de Janeiro, Brasil \\ Universidade Federal Fluminense, Brasil \\ E-mail: gisellegoncalves.farma@gmail.com
}

\begin{abstract}
Resumo
A transfusão sanguínea ainda é o único método de escolha para algumas patologias. Atualmente, mesmo com todo investimento, a hemotransfusão ainda pode trazer riscos ao paciente. O objetivo do estudo foi demonstrar se a educação permanente em saúde está sendo aplicada no setor de hemoterapia, tanto no âmbito nacional ou internacional. Trata-se de uma revisão integrativa, de caráter descritivo e exploratório, através da busca nas bases de dados PubMed, BVS e CAPES. Foram encontrados 207 artigos e selecionados 3, após a aplicação dos critérios de exclusão para a leitura completa e síntese. Dois estavam em inglês e 1 estava em português. Os anos de publicação foram 2013, 2016 e 2019. Quanto aos profissionais pesquisados, 2 artigos abordavam enfermeiros e 1 abordava equipe multiprofissional. Todos os artigos tinham o objetivo de aumentar a segurança transfusional, porém não contemplavam todos os profissionais envolvidos do setor. Os profissionais relatam a necessidade de políticas educativas, porém expressam a preferência por políticas que envolvam situações do cotidiano e que sejam ativas. Embora a educação permanente em saúde envolva aprendizagem significativa e seus benefícios já sejam conhecidos, a literatura não aborda o tema diretamente e poucos foram os artigos relacionados a esse tipo de aprendizagem. Ficou aparente que os programas de treinamentos relacionados a transfusão devam passar por mudanças. Aprendizagem onde há envolvimento da equipe e situações cotidianas aumenta a aprendizagem, aumentado a qualidade do serviço. A melhoria educacional deve ser um processo contínuo e permanente.
\end{abstract}

Palavras-chave: Transfusão sanguínea; Educação continuada; Ensino.

\begin{abstract}
Blood transfusion is still the only method of choice for some pathologies. Currently, even with all investment, blood transfusion can still bring risks to the patient. The aim of the study was to demonstrate whether continuing education in health is being applied in the hemotherapy sector, both nationally and internationally. It is an integrating review, of a descriptive and exploratory nature, through the search in the PubMed, BVS and CAPES databases. 207 articles were found and 3 selected, after applying the exclusion criteria for the complete reading and synthesis. Two were in English and one was in Portuguese. The years of publication were 2013, 2016 and 2019. As for the professionals surveyed, 2 articles addressed nurses and 1 addressed a multidisciplinary team. All articles were intended to increase transfusion safety, but did not include all professionals involved in the sector. Professionals report the need for educational policies, but express a preference for policies that involve everyday situations and are active. Although permanent health education involves significant learning and its benefits are already known, the literature does not address the topic directly and there were few articles related to this type of learning. It became apparent that training programs related to transfusion must undergo changes. Learning where there is team involvement and everyday situations increases learning, increasing the quality of service. Educational improvement must be a continuous and permanent process.
\end{abstract}

Keywords: Blood transfusion; Continuing education; Teaching. 


\section{Resumen}

La transfusión de sangre sigue siendo el único método de elección para algunas patologías. Actualmente, incluso con toda la inversión, las transfusiones de sangre aún pueden traer riesgos para el paciente. El objetivo del estudio fue demostrar si la educación continua en salud se está aplicando en el sector de la hemoterapia, tanto a nivel nacional como internacional. Se trata de una revisión integrativa, de carácter descriptivo y exploratorio, mediante la búsqueda en las bases de datos PubMed, BVS y CAPES. Se encontraron 207 artículos y se seleccionaron 3, luego de aplicar los criterios de exclusión para la lectura completa y síntesis. Dos estaban en inglés y uno en portugués. Los años de publicación fueron 2013, 2016 y 2019. En cuanto a los profesionales encuestados, 2 artículos se dirigieron a enfermeras y 1 a un equipo multidisciplinario. Todos los artículos estaban destinados a aumentar la seguridad de las transfusiones, pero no incluyeron a todos los profesionales involucrados en el sector. Los profesionales informan la necesidad de políticas educativas, pero expresan preferencia por políticas que involucran situaciones cotidianas y son activas. Si bien la educación permanente en salud implica aprendizajes significativos y sus beneficios ya son conocidos, la literatura no aborda el tema de manera directa y existen pocos artículos relacionados con este tipo de aprendizajes. Se hizo evidente que los programas de formación relacionados con la transfusión deben sufrir cambios. Aprender donde hay participación en equipo y situaciones cotidianas aumenta el aprendizaje, aumentando la calidad del servicio. La mejora educativa debe ser un proceso continuo y permanente.

Palabras clave: Transfusión de sangre; Educación continua; Ensenãnza.

\section{Introdução}

Os benefícios da transfusão sanguínea são conhecidos desde o século XIX, porém com muitas reações adversas (Brasil, 2007). A medicina transfusional evolui muito, podendo sua história ser dividida em dois períodos: um que vai até 1900, empírico, e outro a partir de 1900, científico. A era científica foi marcada pela descoberta dos grupos sanguíneos por Karl Landsteiner. Essa descoberta foi base para a medicina moderna relacionada à prática de transfusão de sangue (Flausino et al, 2015).

Em 1901, foi publicada a descoberta de Landsteiner, que classificou o sangue em três grupos de acordo com suas propriedades de aglutinação. Ele estava trabalhando no Departamento de Anatomia Patológica da Universidade de Viena neste momento. Landsteiner cruzou seu soro a glóbulos vermelhos de seis cientistas: cinco colegas de trabalho e ele próprio. Ele descobriu que nenhum dos soros reagiu com seus próprios glóbulos vermelhos, mas o soro do Dr. Pletsching reagiu com as células do Dr. Sturly e o soro de Sturly reagiu com as células de Pletsching. Sugerindo a existência de pelo menos duas classes de anticorpos envolvidos, o que Landsteiner determinou de anti-A e anti-B (

Atualmente, a hemoterapia consiste em uma das alternativas mais eficientes para o tratamento de patologias específicas e para a reposição de derivados sanguíneos e hemocomponentes (Pereira \& Ribeiro, 2014).

Para a segurança de quem receberá a transfusão, todos os hemocomponentes produzidos só serão liberados para consumo, após a realização dos testes imunohematológicos e sorológicos exigidos por legislação específica. Após essa etapa, serão realizadas as provas pré-transfusionais. Essas provas asseguram a compatibilidade sanguínea do doador com o receptor, minimizando os riscos (Brasil, 2017).

Mesmo com todo o investimento feito para a realização de uma transfusão sanguínea segura, esse procedimento não está isento de riscos. A indicação de transfusão de hemocomponentes deve ser baseada em evidências, sendo realizada somente quando não for possível mais nenhuma intervenção terapêutica (Brasil, 2015; Brasil, 2017).

As reações adversas ocasionadas pela hemotransfusão são classificadas como imediatas ou tardias. As complicações imediatas são situações emergenciais que podem ocasionar prejuízos severos aos pacientes, onde o mais sério levaria ao óbito (Carneiro, 2017).

Segundo a Organização Mundial da Saúde (2019), a transfusão de hemocomponentes é composta por várias etapas, desde a triagem de doadores, coleta e processamento, exames sorológicos e provas imunohematológicas, liberação de sangue compatível, até a transfusão do paciente, podendo haver falha em todas elas. Para que o risco na transfusão sanguínea diminua deve-se focar na qualidade do serviço, na educação e treinamento para funcionários. 
Na medicina transfusional, profissionais competentes e bem treinados tornam-se requisitos primordiais para a prevenção de possíveis complicações e reações transfusionais. Estudos demonstram que uma das causas que podem acarretar diminuição da segurança transfusional e prejuízos aos pacientes é a falta de conhecimento técnico e prático pelos profissionais de hemoterapia (Silva, Soares \& Iwamoto, 2009).

Uma das propostas para haver transformação das práticas do trabalho é a Educação Permanente em Saúde (EPS), que utiliza o aprendizado significativo. A aprendizagem significativa se dá através da problematização dos processos de trabalho, trazendo sentido ao aprendizado, correlacionado com a prática, com o cotidiano. Possui o objetivo de transformar os funcionários, as práticas e a organização da instituição, voltada para a realidade local (Arruda et al, 2008).

Para praticar a EPS deve-se constantemente rever o cotidiano e transformá-lo em um local de questionamentos, de produção de subjetividade, de problematização, deve-se produzir e não se conformar (Lemos, 2016).

Existe uma grande diferença entre a educação permanente e a educação continuada, essa última seria a forma tradicional de educar, como Paulo Freire nomeou de "educação bancária", onde o educador "transfere" os conteúdos aos educandos. Visão que torna os homens passivos e sem criatividade (Freire, 2005).

Dessa forma, este estudo foi direcionado pela seguinte questão norteadora: existem evidências na literatura científica nacional e internacional associando o uso da educação permanente em saúde ao setor de hemoterapia com o intuito de aumentar a segurança da prática transfusional?

Diante do exposto, o objetivo deste trabalho, foi demonstrar se a educação permanente em saúde está sendo aplicada no setor de hemoterapia, tanto no âmbito nacional ou internacional.

\section{Metodologia}

Visando responder à questão norteadora, o presente estudo utilizou o método de revisão integrativa da literatura. Segundo Botelho, Cunha e Macedo (2011), "esse método de pesquisa objetiva traçar uma análise sobre o conhecimento já construído em pesquisas anteriores sobre um determinado tema. A revisão integrativa possibilita a síntese de vários estudos já publicados, permitindo a geração de novos conhecimentos, pautados nos resultados apresentados pelas pesquisas anteriores."

Este método permite a tomada de decisão e a melhoria da prática clínica através da análise de pesquisas relevantes, produzindo uma síntese do que existe de conhecimento de determinado tema, além de revelar lacunas do conhecimento que ainda precisam ser preenchidas, direcionando futuras pesquisas (Moura et al, 2018).

O referencial metodológico adotado baseou-se em seis etapas: 1- identificação do tema e seleção da hipótese ou questão de pesquisa para a elaboração da revisão integrativa; 2-estabelecimento de critérios para inclusão e exclusão de estudos/amostragem ou busca na literatura; 3-definição das informações a serem extraídas dos estudos selecionados/categorização dos estudos; 4-avaliação dos estudos incluídos na revisão integrativa; 5-interpretação e discussão dos resultados; 6-apresentação da revisão (Mendes, Silveira \& Galvão, 2018).

Como educação permanente não se encontra inserido como descritor, foi utilizado o descritor educação continuada.

A busca dos artigos foi realizada nas bases de dados: Public Medline ou Publisher Medline (PubMed), Periódicos CAPE e Biblioteca Virtual em Saúde (BVS). Para a seleção dos estudos foram utilizados os Descritores em Ciências da Saúde (DeCS) "transfusão sanguínea" e "educação continuada" e os Medical Subject Headings (MeSH) "blood transfusion" e "continuing education". Utilizou-se o operador booleano AND.

A coleta de dados ocorreu no mês de novembro de 2020. Definiu-se como critério de inclusão: artigo na íntegra, publicados nos últimos dez anos, estudos de fonte primária, publicados em qualquer idioma e estudos em humanos. Definiramse como critérios de exclusão artigos duplicados nas bases de dados e os que não tinham relevância com o estudo. 


\section{Resultados e Discussão}

A pesquisa inicial, nas bases de dados utilizadas, com os critérios de inclusão e exclusão aplicados, resultou em 207 artigos para avaliação dos títulos. Após essa etapa, das quais 12 publicações foram excluídas pelo motivo de duplicidade e 143 não responderam à questão norteadora, foram selecionadas 52 publicações, para terem os seus resumos lidos, visando responder a temática abordada. Quando o título e/ou resumo não eram esclarecedores, buscava-se o artigo na íntegra, com a intenção de não descartar estudos relevantes. Foram selecionados 3 artigos que responderam à questão norteadora e que puderam fazer parte desta pesquisa. Foi elaborado um fluxograma com o detalhamento da seleção dos artigos (Figura 1).

Figura 1: Fluxo de seleção de artigos da revisão integrativa da Literatura.

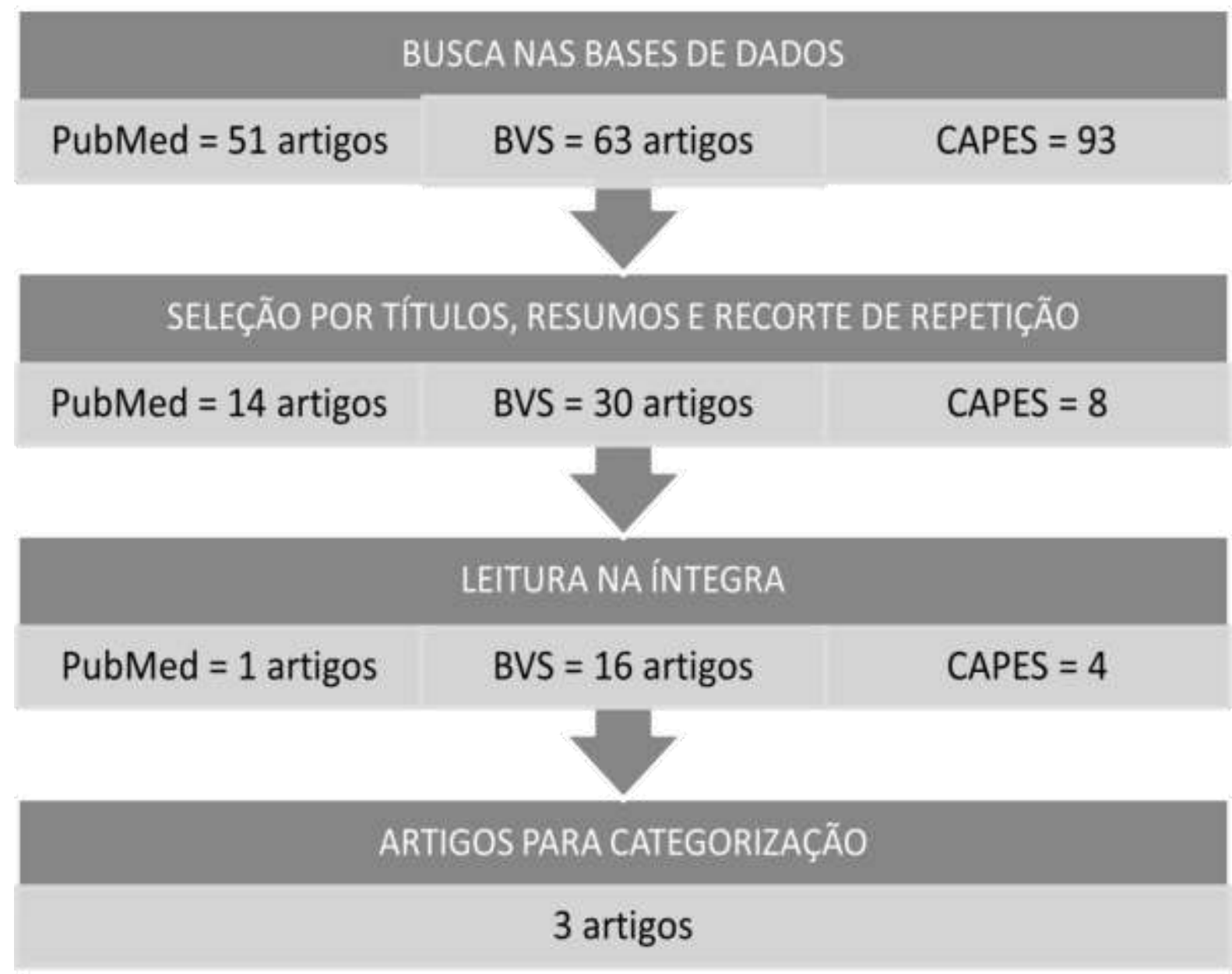

Fonte: Autores.

A terceira etapa foi a categorização dos dados, que consiste na extração, na reunião e na síntese das informações principais dos estudos selecionados. Também tem o intuito de formar um banco de dados com facilidade de acesso (Mendes, Silveira \& Galvão, 2018). Essa revisão integrativa foi composta por 3 publicações, que estão categorizadas no Quadro 1. 
Quadro 1 - Categorização dos artigos selecionados.

\begin{tabular}{|c|c|c|c|c|c|c|c|c|}
\hline \multicolumn{9}{|c|}{ Categorização dos artigos selecionados } \\
\hline Número & $\begin{array}{c}\text { Periódico/ } \\
\text { Base de } \\
\text { dados }\end{array}$ & Título & País & $\begin{array}{l}\text { Sujeitos do } \\
\text { estudo }\end{array}$ & $\begin{array}{c}\text { Autor(res)/ } \\
\text { Ano }\end{array}$ & Tipo de pesquisa & Objetivos & Principais resultados encontrados \\
\hline 1 & $\begin{array}{l}\text { Rev enferm } \\
\text { UFPE on } \\
\text { line. } \\
\text { CAPES }\end{array}$ & $\begin{array}{lr}\text { Educação } & \\
\text { permanente } & \text { de } \\
\text { equipe } & \text { de } \\
\text { enfermagem } & \text { em } \\
\text { reação } & \\
\text { transfusional } & \end{array}$ & Brasil & Enfermeiros & $\begin{array}{l}\text { Nazário, S.S., } \\
\text { Barancelli, } \\
\text { M.D.C., } \\
\text { Gandolfi, M., } \\
\text { Marcondes, } \\
\text { C., Spagnolo, } \\
\text { L.M.L. } \\
\text { (2019) }\end{array}$ & $\begin{array}{l}\text { Qualiquantitativo } \\
\text { e explicativo }\end{array}$ & $\begin{array}{l}\text { Identificar o conhecimento dos } \\
\text { profissionais de Enfermagem } \\
\text { sobre a reação transfusional, antes } \\
\text { e após a aplicação de uma } \\
\text { atividade de educação } \\
\text { permanente. }\end{array}$ & $\begin{array}{l}\text { Quanto às avaliações, o percentual de acerto foi de } \\
56 \% \text { no pré-teste, enquanto, no pós-teste, esse } \\
\text { percentual subiu para } 87 \% \text {, uma melhora de } 31 \% \text { no } \\
\text { percentual de acerto. Mostrou-se, pelo desempenho } \\
\text { dos profissionais entre as avaliações do pré e do pós- } \\
\text { teste, que o treinamento em reação transfusional se deu } \\
\text { de maneira satisfatória, pois, de acordo com o teste } \\
\text { estatístico utilizado, ele atingiu } 82 \% \text { da equipe de } \\
\text { Enfermagem do hospital. }\end{array}$ \\
\hline 2 & $\begin{array}{l}\text { Nurse } \\
\text { Education } \\
\text { in Practice. } \\
\text { PUBMED }\end{array}$ & $\begin{array}{l}\text { Exploring the } \\
\text { opinions of } \\
\text { registered nurses } \\
\text { working in a } \\
\text { clinical } \\
\text { transfusion } \\
\text { environment on } \\
\text { the contribution } \\
\text { of e-learning to } \\
\text { personal learning } \\
\text { and clinical } \\
\text { practice: Results } \\
\text { of a small scale } \\
\text { educational } \\
\text { research study }\end{array}$ & Escócia & Enfermeiros & $\begin{array}{l}\text { Cottrell, S., } \\
\text { Donaldson, } \\
\text { J.H. (2013) }\end{array}$ & $\begin{array}{l}\text { Qualitativa rom } \\
\text { base res } \\
\text { princípios da } \\
\text { fenomenologia }\end{array}$ & 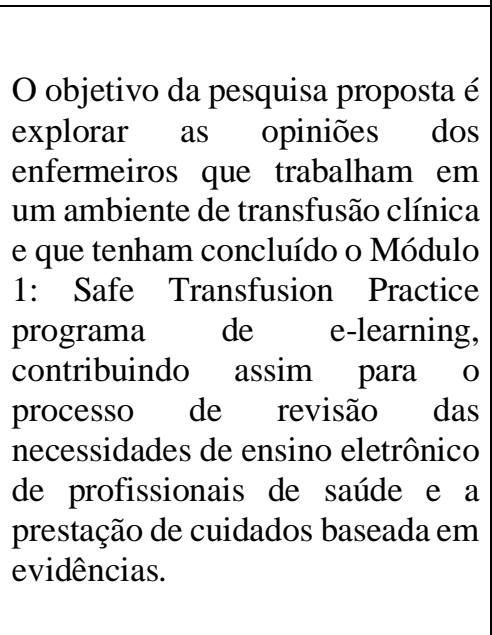 & $\begin{array}{l}\text { Sete participantes da população total da amostra se } \\
\text { ofereceram para participar do estudo. Cinco temas } \\
\text { emergiram que incluíam: as referências de } \\
\text { aprendizagem, aprendizagem interativa, projeto claro, } \\
\text { segurança do paciente e necessidades de aprendizagem } \\
\text { futuras. Os resultados mostram positivamente o } \\
\text { programa de e-learning, os estilos e necessidades de } \\
\text { aprendizagem dos alunos. Em uma tentativa de fazer a } \\
\text { ponte da lacuna prática de conhecimento, outras } \\
\text { opiniões são oferecidas sobre o design do curso e a } \\
\text { aplicação de conhecimento para praticar após a } \\
\text { conclusão do curso. }\end{array}$ \\
\hline
\end{tabular}


Research, Society and Development, v. 10, n. 1, e38510111818, 2021

(CC BY 4.0) | ISSN 2525-3409 | DOI: http://dx.doi.org/10.33448/rsd-v10i1.11818

\begin{tabular}{|c|c|c|c|c|c|c|c|c|}
\hline 3 & $\begin{array}{l}\text { Official } \\
\text { Journal of } \\
\text { the British } \\
\text { Blood } \\
\text { Transfusion } \\
\text { Society. } \\
\text { BVS }\end{array}$ & 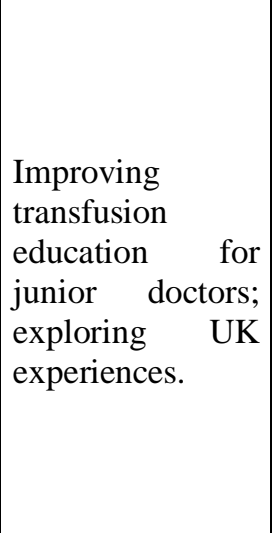 & $\begin{array}{l}\text { Reino } \\
\text { Unido }\end{array}$ & $\begin{array}{l}\text { Médicos } \\
\text { residentes e } \\
\text { profissionai } \\
\text { s de saúde }\end{array}$ & $\begin{array}{l}\text { Graham, } \\
\text { J.E., Narayan, } \\
\text { S., Pendry, K. } \\
\text { (2016) }\end{array}$ & Qualitativa & $\begin{array}{l}\text { Fornecer orientação baseada em } \\
\text { evidências sobre como a } \\
\text { educação transfusional deve ser } \\
\text { entregue aos médicos residentes, } \\
\text { empregando metodologia de } \\
\text { pesquisa qualitativa estabelecida. }\end{array}$ & $\begin{array}{l}\text { Oito grupos focais foram formados envolvendo } 53 \\
\text { funcionários, } 77 \% \text { dos quais eram médicos. A atual } \\
\text { educação transfusional para os médicos no Reino } \\
\text { Unido é dependente de e-learning e 'formação cascata' } \\
\text { (no trabalho de clínicos seniores / pessoal de } \\
\text { enfermagem). E-learning é visto como uma "caixa } \\
\text { exercício". Há uma chamada para a educação } \\
\text { continuada relevante fornecida por bons educadores } \\
\text { em um ambiente longe da prática clínica. Métodos } \\
\text { preferidos incluem pequeno grupo e aprendizagem de } \\
\text { simulação baseado em casos da vida real. Em contraste } \\
\text { com competência prática, a avaliação clínica de } \\
\text { competência é considerada inviável. }\end{array}$ \\
\hline
\end{tabular}

Fonte: Autores. 
Dos artigos utilizados para a revisão 2 (67\%) estavam em inglês e 1 (33\%) estava em português. Os anos das publicações foram 2013, 2016 e 2019. Quanto aos profissionais pesquisados 2 (67\%) artigos abordavam enfermeiros, 1 (33\%) abordava equipe multidisciplinar.

As pesquisas tiveram diferentes objetivos: avaliar, identificar e explorar as diferentes formas de educação voltadas para a segurança da transfusão sanguínea. Os estudos demonstraram as necessidades educacionais relacionadas aos profissionais envolvidos na transfusão sanguínea.

Cada vez mais a transfusão sanguínea vem sendo desestimulada por diversos autores, isto porque seu uso pode trazer riscos para o paciente, variando entre reações adversas leves até as mais graves, como a morte. Porém, em muitos casos, ela ainda se faz necessária, não havendo outro recurso terapêutico (Nazario et al, 2019).

Segundo o mesmo autor, muitos profissionais que atuam no setor de hemoterapia, mesmo estando capacitados, sentemse inseguros para tomar decisões diante dos efeitos adversos causados pela transfusão sanguínea.

Estudos relataram que, mesmo após treinamentos específicos sobre a prática segura de transfusão, o conhecimento dos participantes diminui ao longo do tempo, sendo necessárias práticas de aprendizagem constantes (Smith et al 2013).

Graham, Naraya e Pendry (2016) destacam que para a prática de uma transfusão ideal, deve-se realizar melhorias na infraestrutura dos programas de transfusão, rastreio de doenças infecciosas, além do processo de doação; mas a educação dos profissionais de saúde envolvidos é fundamental para que isso ocorra. Médicos e pesquisadores repetidamente solicitam mais educação em medicina de transfusão.

A educação caracteriza-se como método eficaz para tornar a prescrição de componentes sanguíneos mais criteriosa, diminuindo a sua utilização, em consequência, aumentando a segurança do paciente (Sajwani, 2012; Corwin et al, 2014).

Destaca-se o fato de que muitas vezes o uso inadequado de hemocomponentes pode aumentar a probabilidade das reações adversas, devendo -se procurar métodos alternativos para evitar o seu uso, quando possível.

Amaral et al (2016) relataram em seu estudo que existe uma fragilidade em relação aos conhecimentos dos profissionais envolvidos no ato da transfusão, devendo ser aplicada não só a educação continuada, como também a educação permanente a esses profissionais.

Nazário et al (2019) pontuaram que a educação permanente qualifica a assistência à saúde tornando os profissionais mais aptos a prevenir o surgimento de reações transfusionais graves, garantindo uma maior segurança ao paciente.

Em países desenvolvidos, a educação permanente já é aplicada há 20 anos na graduação, porém ainda existem poucos relatos no que diz respeito aos profissionais de saúde. Metodologias educativas ativas, críticas e reflexivas sempre foram muito valorizadas nos países desenvolvidos, não sendo muito utilizadas no ambiente profissional (Pereira et al 2017).

Cottrell e Donaldson (2013) identificaram que existe uma lacuna entre a teoria e a prática na educação transfusional. Para a teoria ser compreendida existe a necessidade de ser demonstrada.

Os modelos de educação em saúde, no decorrer dos anos, estão sofrendo mudanças, baseados, muitas vezes, na interação educando e educador, levando em consideração os saberes do profissional, com base na reflexão, consciência e autonomia (Coriolano et al, 2012).

No quadro 1 , o artigo número 3 , demonstrou que os médicos relataram haver necessidade de políticas educativas voltadas para os problemas que possam surgir no cotidiano e a aprendizagem deveria ser realizada em pequenos grupos. O estudo também demonstrou a preferência dos participantes pela educação em cenários reais, onde possa haver a ligação da prática com a teoria aprendida (Graham, Naraya \& Pendry 2016).

Para obter impacto no processo de aprendizagem, educadores de saúde devem realizar adaptações nos processos de educação, atendendo as diversas necessidades de aprendizagem dos profissionais de saúde. Os educadores clínicos devem trabalhar para que os profissionais de saúde se tornem habilidosos e competentes na sua função (Cottrell \& Donaldson, 2013). 
Para que ocorra uma aprendizagem significativa, isto é, onde haverá mudança de comportamento, a prática pedagógica utilizada pelo educador deverá tornar-se o profissional um sujeito ativo, crítico e autônomo. Sendo capaz de aprimorar-se não só, no ambiente de trabalho como na sociedade (Peixoto, 2016).

\section{Conclusão}

Os riscos inerentes a uma transfusão são diversos, podendo ir de leves até fatais, por isso profissionais envolvidos com esse processo necessitam de treinamentos constantes e aprendizados significativos. Todas as etapas da transfusão necessitam de atenção, desde a captação de doadores de sangue, até a transfusão propriamente dita, garantindo com isso uma maior qualidade do serviço prestado.

Ficou comprovado que, com o passar do tempo, os aprendizados são esquecidos e muitos profissionais tornam-se inseguros. Também foi demonstrado que os profissionais necessitam de maior educação sobre o assunto.

A Educação Permanente, apesar de ser muito importante para a prática do trabalho, ainda é pouco utilizada no setor de hemoterapia. Somente um trabalho abordou diretamente o tema e os outros dois de maneira indireta, embora, todos citem a educação como um aspecto muito importante para a segurança transfusional.

Os estudos não abrangeram todos os funcionários que compõem o setor de hemoterapia, pois, além dos enfermeiros e médicos, existem ainda, os profissionais responsáveis pelas provas de compatibilidade sanguínea. Uma equipe multiprofissional que possui interação e recebe educação para a melhoria das práticas do trabalho é capaz de ofertar um serviço de maior qualidade.

Ficou aparente que os programas de treinamento relacionados a transfusão, que existem atualmente, devam ser modificados. De acordo com os profissionais que participaram das pesquisas, metodologias ativas que exijam maior interação entre os participantes e utilizem situações do cotidiano seriam mais eficazes. Foi evidenciado que onde ocorre maior valorização do conhecimento prévio e das sugestões dadas pela equipe envolvida no processo de trabalho, há um maior aprendizado.

Esforços para a melhoria educacional em hemoterapia precisam ser colaborativos e inovadores, envolvendo, não só, uma equipe multiprofissional de especialistas em medicina transfusional, mas também em educação. Devendo ser um processo contínuo e permanente.

Como ficou comprovado, o tema do estudo não está esgotado na literatura científica nacional e internacional. Sugerese que educadores e profissionais de hemoterapia, embasados por esse estudo, levem para o ambiente de trabalho a educação permanente; divulgando, através de trabalhos científicos, como se deu a implantação e os resultados obtidos.

\section{Referências}

Amaral, J. H. S., Nunes, R. L. S., Rodrigues, L. M. S., Braz, M. R, Balbino, C. M. \& Silvino, Z. R. (2016). Hemoterapia: um desafio no cotidiano da equipe de enfermagem. Rev Enferm UFPE Online. 2016; 10(6):4820-4827. https://periodicos.ufpe.br/revistas/revistaenfermagem/article/view/11261

Arruda, M. P., Araújo, A. P., Locks, G. A. \& Pagliosa, F. L. (2008). Educação permanente: uma estratégia metodológica para os professores da saúde. Revista Brasileira de Educação Médica, 32(4), 518-524. https://www.scielo.br/scielo.php?script=sci_arttext\&pid=S0100-55022008000400015\&lng=pt\&tlng=pt.

Botelho, L. L. R.; Cunha, C. C. de A.; Macedo, M. (2011). O método da revisão integrativa nos estudos organizacionais. Rev. Eletrônica Gestão e Sociedade. 5(11), 121-136 ISSN 1980-5756. http://biblioteca.cofen.gov.br/wp-content/uploads/2019/06/manual_revisao_bibliografica-sistematica-integrativa.pdf

Brasil (2007). Hemovigilância: manual técnico para investigação das reações transfusionais imediatas e tardias não infecciosas. Agência Nacional de Vigilância Sanitária. Brasília. https://www.gov.br/anvisa/pt-br/assuntos/fiscalizacao-e-monitoramento/hemovigilancia/publicacoes/manual-tecnico-de-hemovigilanciainvestigacao-das-reacoes-transfusionais-imediatas-e-tardias-nao-infecciosas.pdf/view .

Brasil (2015). Guia para uso de hemocomponentes. (2a ed.). Ministério da saúde. Brasília. https://bvsms.saude.gov.br/bvs/publ icacoes/guia_uso_hemocomponentes_2ed.pdf.

Brasil (2017). Portaria de Consolidação $n^{\circ} .5$, de 28 de setembro de 2017. Determina o Regulamento Técnico para os procedimentos hemoterápicos, incluindo a coleta, o processamento, a testagem, o armazenamento, o transporte, o controle de qualidade e o uso humano de sangue, e seus componentes, obtidos do sangue venoso, do cordão umbilical, da placenta e da medula óssea. Ministério da Saúde. Brasília. http://bvsms.saude.gov.br/bvs/saudelegis/gm/2017/prc0005_03_10_2017.html. 
Coriolano, M. W. L., Lima, M. M., Queiroga, B. A. M., Ruiz-Moreno, L. \& Lima, L. S. (2012). Educação permanente com agentes comunitários de saúde: uma proposta de cuidado com crianças asmáticas. Trabalho, Educação e Saúde, 10(1), 37-59. https://www.scielo.br/scielo.php?script=sci_arttext\&pid=S198177462012000100003

Corwin, H.L., Theus, J. W., Cargile, C.S. \& Lang, N.P. (2014). Red blood cell transfusion: impact of an education program and a clinical guideline on transfusion practice. Journal of hospital medicine, 9(12), 745-749. https://doi.org/10.1002/jhm.2237.

Cottrell, S. \& Donaldson, J.H. (2013). Exploring the opinions of registered nurses working in a clinical transfusion environment on the contribution of e-learning to personal learning and clinical practice: results of a small scale educational research study. Nurse education in practice, 13(3), 221-227. https://doi.org/10.1016/j.nepr.2013.01.014.

Flausino, G. F., Nunes, F. F., Cioff, J. G. M. \& Carneiro-Proietti, A. B. F. (2015). O ciclo de produção do sangue e a transfusão: o que o médico deve saber. Rev Med Minas Gerais, 25 (2), 269-279. http://www.rmmg.org/artigo/detalhes/1784.

Freire, P. (2005). Pedagogia do oprimido. Paz e Terra. (46a ed.).

Graham, J.E., Narayan, S. \& Pendry, K. (2017). Improving transfusion education for junior doctors; exploring UK experiences. Transfusion medicine (Oxford, England), 27(2), 96-104. https://doi.org/10.1111/tme.12373.

Lemos, C. L. S. (2016). Educação Permanente em Saúde no Brasil: educação ou gerenciamento permanente? Ciência \& Saúde Coletiva, 21(3), 913922. https://www.scielo.br/scielo.php?pid=S1413-81232016000300913\&script=sci_abstract\&tlng=pt

Mendes, K. D. S., Silveira, R. C. C. P. \& Galvão, C. M. (2008). Revisão integrativa: método de pesquisa para a incorporação de evidências na saúde e na enfermagem. Texto \& Contexto - Enfermagem, 17(4), 758-764. https://doi.org/10.1590/S0104-07072008000400018.

Moura, L. R., Torres, L. M., Cadete, M. M. M. \& Cunha, C. F. (2018). Fatores associados aos comportamentos de risco à saúde entre adolescentes brasileiros: uma revisão integrativa. Revista da Escola de Enfermagem da USP, 52, e03304. https://doi.org/10.1590/s1980-220x2017020403304.

Nazário, S., Marcondes, C., Barancelli, M., Gandolfi, M. \& Spagnolo, L. (2019). Educação permanente de equipe de enfermagem em reação transfusional. Revista de Enfermagem UFPE on line, 13(2), 307-314. https://doi.org/10.5205/1981-8963-v13i2a237361p307-314-2019.

Organização Mundial Da Saúde. https://www.who.int/bloodsafety/quality/en/.

Peixoto, A. G. (2016). O uso de metodologias ativas como ferramenta de potencialização da aprendizagem de diagramas de caso de uso. Periódico científico outras palavras, 12(2), 35-50. http://revista.faculdadeprojecao.edu.br/index.php/Projecao5/article/view/718/604.

Pereira, A. L. \& Ribeiro, M. C. P. (2014). Terapias alternativas às transfusões de sangue. Revista da Universidade Vale do Rio Verde, 12 (2), 566-579. http://periodicos.unincor.br/index.php/revistaunincor/article/view/1597.

Pereira, S., Capelli, J., Abrahão, A. \& Anastacio, A. (2017). A experiência do uso da aprendizagem baseada em projetos como metodologia ativa no programa de educação pelo trabalho para a saúde na aprendizagem da prática profissional. DEMETRA: Alimentação, Nutrição \& Saúde, 12(4), 881-898. https://doi.org/10.12957/demetra.2017.28092.

Sajwani, F.H. (2012). Improving blood transfusion practice by regular education in the United Arab Emirates. Transfusion,52(7 Pt 2), 1628-1631. https://doi.org/10.1111/j.1537-2995.2012.03760.x.

Schwarz, H. P. \& Dorner, F. (2003). Karl landsteiner and his major contributions to haematology. British Journal of Haematology, 121 (4), 556-565. https://pubmed.ncbi.nlm.nih.gov/12752096/.

Silva, K. F. N., Soares, S. \& Iwamoto, H.H. (2009). A prática transfusional e a formação dos profissionais de saúde. Revista Brasileira de Hematologia e Hemoterapia, 31(6), 421-426. https://www.scielo.br/scielo.php?pid=S1516-84842009005000092\&script=sci_abstract\&tlng=pt.

Smith, A., Gray, A., Atherton, I., Pirie, E. \& Jepson, R. (2014). Does time matter? An investigation of knowledge and attitud es following blood transfusion training. Nurse education in practice, 14(2), 176-182. https://doi.org/10.1016/j.nepr.2013.08.016. 